\title{
On a Relation Between Two-Dimensional Fourier Integrals and Series of Hankel Transforms
}

\author{
J. V. Cornacchio* and R. P. Soni*
}

(June 1, 1965)

\begin{abstract}
Procedures are developed for expressing two-dimensional Fourier transforms in terms of tabulated one-dimensional transforms.
\end{abstract}

In the theoretical solution recently obtained for stationary spatial-coherence functions over radiating apertures, [1] ${ }^{1}$ the evaluation of the two-dimensional Fourier integral of the far-field intensity distribution is required. Since the appearance of such integrals is also quite common in other areas of mathematical physics, it would be useful to render their evaluation amenable to the application of extensively tabulated results available in the literature. (For examples of such sources see [2], [4], [5], and [6].) For functions of one variable, comprehensive tables of Fourier transforms exist [2], and although it is possible to reduce the $k$ dimensional Fourier transform of radial functions $^{2}[3]$ to Hankel transforms [3, p. 69] for which extensive tables [5] are available, there are no tables giving the Fourier transform for $k>1$ of arbitrary functions (i.e., nonradial) even in the case of $k=2$. In this paper, the two-dimensional Fourier transform is reduced to a form which facilitates its evaluation by the use of existing tables $[4,5]$ and also yields a result which is an extension of that given in Bochner and Chandrasekharan [3], for the case $k=2$, to functions which are not necessarily radial. It will be shown that if $g(\alpha, \beta)$ is the two-dimensional Fourier transform of $f(x, y)$, i.e.,

$$
g(\alpha, \beta)=\int_{-\infty}^{\infty} \int_{-\infty} f(x, y) e^{i(\alpha x+\beta y)} d x d y
$$

then, if $f$ is sufficiently well behaved, it is possible to express $g(\alpha, \beta)$ in the following form:

$$
g(\alpha, \beta)=\sum_{n=0}^{\infty} a_{n}(\alpha, \beta) h_{n}\left\{A_{n} ; \sqrt{\alpha^{2}+\beta^{2}}\right\}
$$

*IBM Systems Development Division, Endicott, N.Y

${ }^{1}$ Figures in brackets indicate the literature references at the end of this paper.

${ }^{2} f\left(x_{1}, x_{2}, \ldots, x_{k}\right)$ is a radial function if it can be written in the form

$$
f\left(x_{1}, x_{2}, \ldots, x_{k}\right)=F\left(\sqrt{x_{1}^{2}+x_{2}^{2}+\ldots+x_{k}^{2}}\right)
$$

where

$$
h m\{g ; z\}=\int_{0}^{\infty} \sqrt{z t} g(t) J_{m}(z t) d t ;
$$

$A_{n}$ are certain functions associated with $f(x, y)$, and $a_{n}(\alpha, \beta)$ are functions independent of $f$. It may be noted that $h_{m}\{g ; z\}$ is the Hankel transform of order $m$ of $g(t)$.

The derivation of (2) proceeds by rewriting (1) in the form

$$
g(\alpha, \beta)=\int_{r=0}^{\infty} \int_{\theta=0}^{2 \pi} f(r, \theta) e^{i(\alpha r \cos \theta+\beta r \sin \theta)} r d r d \theta .
$$

Assuming that $f(r, \theta)$ can be represented by the following Fourier series

$$
f(r, \theta)=\sum_{n=-\infty}^{\infty} f_{n}(r) e^{-i n \theta},
$$

where

$$
f_{n}(r)=\frac{1}{2 \pi} \int_{0}^{2 \pi} f(r, \theta) e^{i n \theta} d \theta,
$$

and that the interchange of summation and integration is valid, (4) can be written as

$$
g(\alpha, \beta)=\sum_{n=-\infty}^{\infty} \int_{0}^{\infty} f_{n}(r) r d r \int_{0}^{2 \pi} e^{i(-n \theta+\alpha r \cos \theta+\beta r \sin \theta)} d \theta \text {. }
$$

However, using the well-known integral representation for $J_{n}(z)$, the Bessel function of order $n[6, p$. 14 , eq (2)], it can be shown that

$$
\int_{0}^{2 \pi} e^{i(-n \theta+\alpha r \cos \theta+\beta r \sin \theta)} d \theta=2 \pi e^{i n \tan ^{-1}(\alpha / \beta)}
$$

$$
J_{n}\left(\sqrt{\alpha^{2}+\beta^{2}} r\right)
$$


Thus, using (7), $g(\alpha, \beta)$ can be written

$g(\alpha, \beta)=2 \pi \sum_{n=-\infty}^{\infty} e^{+i n \tan ^{-1}(\alpha / \beta)} \int_{0}^{\infty} f_{n}(r) J_{n}\left(\sqrt{\alpha^{2}+\beta^{2}} r\right) r d r$.

Observing that

$$
J_{-n}(x)=(-1)^{n} J_{n}(x)
$$

and, $f$ being real

$$
f_{-n}(r)=f_{n}^{*}(r)
$$

(8) can be written, (after some simplification)

$g(\alpha, \beta)=\pi \sum_{n=0}^{\infty} \epsilon_{n} \int_{0}^{\infty}\left[C_{n}+(-1)^{n} C_{n}^{*}\right] J_{n}\left(\sqrt{\alpha^{2}+\beta^{2}} r\right) d r$

where

$$
C_{n}=f_{n}(r) e^{i n \tan ^{-1}(\alpha / \beta)},
$$

and

$$
\epsilon_{n}=\left\{\begin{array}{l}
1, n=0 \\
2, n>0 .
\end{array}\right.
$$

This establishes the result (2) where

$$
a_{n}(\alpha, \beta)=\pi \epsilon_{n}\left(\alpha^{2}+\beta^{2}\right)^{-1 / 4}
$$

and

$$
A_{n}=\sqrt{r}\left[C_{n}+(-1)^{n} C_{n}^{*}\right] .
$$

For functions of the form

$$
f(r, \theta)=F(r)\left\{\begin{array}{l}
\cos m \theta \\
\sin m \theta
\end{array}\right\}, m \text { an integer, }
$$

the right-hand side of (9) reduces to the single term

$$
\begin{gathered}
g(\alpha, \beta)=\frac{\pi \epsilon_{m}(i)^{m}}{\left(\alpha^{2}+\beta^{2}\right)^{1 / 4}} h_{m}\{\sqrt{r} F(r) ; \\
\left.\sqrt{\alpha^{2}+\beta^{2}}\right\}\left\{\begin{array}{c}
\cos \left[m\left(\pi / 2-\tan ^{-1}(\alpha / \beta)\right)\right] \\
m=0,1,2, \ldots \\
\sin \left[\begin{array}{c}
\left.m\left(\pi / 2-\tan ^{-1}(\alpha / \beta)\right)\right] \\
m=1,2,3, \ldots \ldots
\end{array}\right.
\end{array}\right\}
\end{gathered}
$$

By setting $m=0$ in (13), one obtains

$$
g(\alpha, \beta)=2 \pi\left(\alpha^{2}+\beta^{2}\right)^{-1 / 4} h_{0}\left\{\sqrt{r} F(r) ; \sqrt{\alpha^{2}+\beta^{2}}\right\}
$$

which shows that, for $f(r, \theta)=F(r), g(\alpha, \beta)$ is a radial function. This special case of (13) yields the relation between a radial function and its Fourier transform which is given in [3, p. 69].

Finally, due to the relation $[5$, p. 3$]$

$\mathscr{L}\left\{t^{1 / 2 \nu-1 / 4} h_{i}\left\{f ;(2 t)^{1 / 2}\right\} ; s\right\}$

$$
=s^{-\nu-1} \mathscr{L}\left\{t^{1 / 2 \nu-1 / 2} f\left[(2 t)^{1 / 2}\right] ; s^{-1}\right\}
$$

between Hankel and Laplace transforms, the extensive tables of Laplace and inverse Laplace transforms [4] can be used to evaluate Hankel transforms. Hankel transforms may also be obtained by methods given in $[4,5]$. These observations make the results given here quite comprehensive in their application.

The authors would like to acknowledge the assistance of T. C. Ku of this laboratory during the development of the material reported here.

\section{Added Note Concerning Bochner's Theorem on Radial Functions}

A paper has recently been published by C. Bollini et al., (Journal of Mathematical Physics, Vol. 6, p. $165,1965)$ in which Bochner's result for $k=4$ has been generalized to include "casual distributions," that is they show that if $\psi\left(y_{1}, y_{2}, y_{3}, y_{4}\right)$ is a causal radial distribution and $I\left(q_{1}, q_{2}, q_{3}, q_{4}\right)$ is its 4-dimensional Fourier transform, then $I$ is a radial function. In particular,

$$
I\left(q_{1}, q_{2}, q_{3}, q_{4}\right) \equiv I\left(q^{2}\right)=\frac{4 \pi^{2}}{i q} \int_{0}^{\infty} \psi\left(R^{2}\right) J_{1}(q R) R^{2} d R
$$

where:

$$
\begin{aligned}
& R=\left[y_{1}^{2}+y_{2}^{2}+y_{3}^{2}+y_{4}^{2}\right]^{1 / 2} \\
& q^{2}=\left[q_{1}^{2}+q_{2}^{2}+q_{3}^{2}+q_{4}^{2}\right]^{2}
\end{aligned}
$$

\section{References}

[1] J. V. Cornacchio and R. P. Soni, J. Opt. Soc. Am. 55, (1965); 107, Il Nuovo Cimento (to be published).

[2] F. Oberhettinger, Tabellen Zur Fourier Transformations (Springer-Verlag, Berlin, 1957).

[3] S. Bochner and K. Chandrasekharan, Fourier Transforms, Annals of Mathematics Study No. 19, Princeton, 1949.

[4] A. Erdelyi et al., Tables of Integral Transforms, I, (McGrawHill Publishing Company, New York, 1954).

[5] A. Erdelyi et al., Tables of Integral Transforms, II, (McGrawHill Publishing Company, New York, 1954).

[6] A. Erdelyi et al., Higher Transcendental Functions, II, (McGraw Hill Publishing Company, New York, 1953).

(Paper 69B3-149) 